\title{
Les Français musulmans : enquête sur une rébellion républicaine
}

\section{Ayhan Kaya}

Traducteur : Aude Aylin de Tapia et Stéphane de Tapia

\section{(2) OpenEdition \\ Journals}

\author{
Édition électronique \\ URL : https://journals.openedition.org/remi/3321 \\ DOI : $10.4000 /$ remi.3321 \\ ISSN : $1777-5418$ \\ Éditeur \\ Université de Poitiers \\ Édition imprimée \\ Date de publication : 31 décembre 2006 \\ Pagination : 135-153 \\ ISBN : 978-2-911627-43-9 \\ ISSN : 0765-0752

\section{Référence électronique} \\ Ayhan Kaya, "Les Français musulmans : enquête sur une rébellion républicaine », Revue européenne \\ des migrations internationales [En ligne], vol. 22 - $n^{\circ} 3$ | 2006, mis en ligne le 31 décembre 2009, consulté \\ le 14 avril 2022. URL : http://journals.openedition.org/remi/3321 ; DOI : https://doi.org/10.4000/remi. \\ 3321
}

Ce document a été généré automatiquement le 14 avril 2022.

(c) Université de Poitiers 


\title{
Les Français musulmans : enquête sur une rébellion républicaine
}

\author{
Ayhan Kaya \\ Traduction : Aude Aylin de Tapia et Stéphane de Tapia
}

Je voudrais exprimer ma reconnaissance à l'Académie turque des Sciences et au Fond de la Recherche de l'Université Bilgi qui m'ont permis de mener mes travaux en France. Je remercie également Clémence Delmas, Joss Dray, Malika Chafi, Dominique Vital, Eric Macé, şirin Dilli, Verda Irtiş, Senem Aydın, Burak Onaran et Stéphane de Tapia pour leur amitié et nos discussions.

\footnotetext{
L'histoire l'enseigne, nos chances sont vaines

Alors arrêtons tout plutôt que c'la traîne, Ou ne draine, même, encore plus de haine.

Unissons-nous pour incinérer ce système. Mais qu'est-ce, mais qu'est-ce qu'on attend pour foutre le feu?

(Suprême NTM, « Qu'est-ce qu'on attend »)1
}

Pendant les émeutes de novembre 2005 en France, beaucoup a été dit sur la montée du radicalisme religieux dans la jeunesse musulmane. Cet article veut montrer que ce ne sont pas tant la différence culturelle et l'islamisme qui ont fait descendre les jeunes musulmans dans la rue qu'une réaction en chaîne à deux siècles de colonialisme et de racisme, renforcés par des phénomènes récents de pauvreté et d'exclusion. Serait-ce une sonnette d'alarme pour le républicanisme français? Dans le contexte occidental européen, les relations de bon voisinage se sont transformées en relations tournant autour des différences religieuses, ethniques et culturelles. On estime qu'en moyenne, dans les pays d'Europe occidentale, un habitant sur dix est immigré ou issu plus généralement d'une population différente de la majorité ethnoculturelle. Au même moment, dans les pays de l'hémisphère sud, seule une personne sur 70 est immigrée. C'est ce qui différencie principalement pays du Nord et du Sud en termes de cohabitation entre résidents voisins, issus de groupes ethnoculturels et religieux divers. Ce type de relation a créé des tensions au fil des siècles entraînant intimidation, 
annihilation, assimilation de certains de ces "voisins». Une telle tension a pu se manifester durant les époques de crises structurelles, sociopolitiques ou socioéconomiques. On sait que la coexistence pacifique et l'absence de tensions peuvent prévaloir pendant les époques de prospérité. Cependant, pendant les périodes de crise économique, et leurs suites politiques et juridiques, nous avons été témoins de nombreux récits où nos "voisins de palier" ont été sujets à des mécanismes d'exclusion, de marginalisation ou de minorisation pour devenir « les Autres » et « les ennemis de l'intérieur». L'un des plus évidents exemples de cette expérience est la manière dont les immigrés de culture musulmane vivant dans les sociétés occidentales sont en train de devenir «les Autres». Les démocraties occidentales qui n'accordent pas de droits politiques suffisants aux immigrés et qui ne les encouragent pas à assurer leur propre représentation politique font aujourd'hui face à quelques dilemmes importants. Il n'est pas surprenant que les États-Nations démocratiques qui ne peuvent surmonter leurs problèmes structurels et, par conséquent, affronter légitimement les crises prennent un raccourci et en reportent la responsabilité sur leurs « hôtes-voisins de palier ». Il est possible d'expliquer la récente et violente révolte vécue en France dans ce cadre.

2 Le problème de l'intégration de la population immigrée a une fois de plus retenu l'attention, suite aux actes de violence qui se sont déroulés dans les banlieues de Paris durant ce mois d'octobre 2005 et dans plusieurs autres villes françaises et quelques villes belges, néerlandaises et même allemandes. Après que deux jeunes d'origines nord-africaine et subsaharienne aient été électrocutés alors qu'ils essayaient de se cacher de la police parisienne dans un transformateur électrique, des milliers de jeunes musulmans, pour la plupart d'origines maghrébines et africaines, sont descendus dans les rues ${ }^{2}$. Comment interpréter ces actes de violence et de haine, surtout venant d'une " deuxième " ou " troisième " génération d'immigrés (très généralement de nationalité française)? Le Président français, Jacques Chirac, laisse alors entendre que ce soulèvement est la conséquence de problèmes sociaux structurels, faisant ainsi une allusion subtile au fait qu'il est conscient du problème, alors que le ministre de l'Intérieur, Nicolas Sarkozy, opte pour une description des jeunes musulmans des banlieues usant de qualificatifs tels que "criminels", "vagabonds", "voyous " et « racaille», et s'efforce de gagner le soutien de l'opinion publique. Ce dernier a même prétendu que ces jeunes musulmans avaient des liens avec des organisations islamistes terroristes telles qu'Al Qaida ${ }^{3}$. Depuis, beaucoup d'hommes politiques et d'intellectuels sont encore loin d'apporter une explication critique et substantielle à cette tension interne à la société. Un dangereux abîme s'est ouvert entre la majorité de la population française, principalement de culture catholique, et les immigrés musulmans dont le nombre approche les cinq millions ${ }^{4}$.

3 Alors que cet événement semble extrême, avec l'incendie de plusieurs milliers de voitures et de dizaines d'entreprises privées, des agressions contre des bâtiments publics, l'aggravation de la tension sociale et la référence à l'identité musulmane, il faut noter que des événements similaires ont été fréquents en France durant les deux dernières décennies. "La Haine ", film de Mathieu Kassowitz (1996), avait déjà particulièrement attiré l'attention dans le monde entier sur la jeunesse immigrée de France. Ce film-culte donne une perspective affûtée et désinhibée de la vie quotidienne de la jeunesse vivant dans les banlieues parisiennes. Il illustre la marginalisation, la solitude, l'absence d'espoir de ces jeunes gens, les problèmes d'éducation, d'emploi, de 
discrimination et de xénophobie auxquels ils font face et brosse un tableau de la culture de la protestation (rap, graffiti, break-danse, hip-hop...). Mathieu Kassowitz soulignait que les jeunes Maghrébins particulièrement, les «Beurs », ont trouvé leur salut dans des mouvements militants islamistes et dans des gangs de rue.

Aussi bien lors des émeutes actuelles que passées (dans les années 1990), l'opinion publique a souligné la radicalisation progressive de l'identité musulmane adoptée par la jeunesse (Bloul, 1998; Hargreaves, 1995 ; Cesari, 2003). Dans la perspective de la montée constante de la popularité de la thèse du «choc des civilisations » d'Huntington, développée en 1992, il devient facile de comprendre pourquoi l'entité musulmane est si fortement interpellée et montée en épingle en Occident. Les politiques identitaires, avec des aspects culturels, ethniques et religieux, sont d'abord produites par des groupes marginaux, minoritaires, d'immigrés dont la majorité appartient aux classes ouvrières et les plus pauvres. On peut faire le parallèle avec les analyses d'Alasdair MacIntyre (1971), pour lequel il y a deux modes de politique : les "politiques des établis» (politics of the insiders) et les «politiques d'étrangers " (politics of the outsiders) $)^{5}$. Les établis portent leur pratique politique via des institutions politiques légitimes (Parlement, partis politiques, médias) alors que les étrangers (outsiders) s'appuient sur la culture, l'ethnie, la religion et les traditions pour atteindre leurs buts (Bloul, 1999). Le développement de pratiques ethnoculturelles et religieuses par les immigrés et les groupes minoritaires peut donc être perçu comme un désir de rattachement à la sphère politico-publique.

On doit garder à l'esprit qu'il y a deux différences fondamentales entre les émeutes récentes et les plus anciennes : d'abord les dernières émeutes ne se sont pas limitées à la capitale et ensuite l'intensité de la violence a été beaucoup plus forte. La première action collective "beur» qui constitue un tournant en termes de visibilité politique correspond à la Marche pour l'Égalité des Droits et contre le Racisme (1983). L'initiative de la marche était une réaction à l'escalade de la violence raciste. Elle était en partie inspirée du modèle non-violent du mouvement des droits civils des États-Unis, dirigé par Martin Luther King. Cette orientation était celle, pour partie des 40 "marcheurs " qui commencèrent leur périple à Marseille pour finir, un mois plus tard, à Paris, accompagnés de quelque 100000 personnes. Les demandes des «Beurs» s'exprimaient en termes moraux, comme la quête pour l'égalité, la dignité et les droits, un État pacifique fondé sur la solidarité (Bloul, 1999; Jelen, 2005). Depuis ses origines, le mouvement beur se divise entre politique électorale et actions sociales locales, en d'autres termes, entre le civique et le civil. Ce qui serait primordial n'est pas encore clair pour les Beurs: revendications civiques ou revendications civiles? On observe des changements dans les discours des émeutiers récents - qui sont les fils des manifestants de 1983 - ; le discours pacifiste «à la Martin Luther King » fait place à un discours plus radical "à la Malcolm-X ", avec une connotation islamique plutôt que républicaine ${ }^{6}$. On peut voir là l'échec de l'élite politique beure des années 1980 . Cet échec et l'aggravation de l'exclusion sociale dans les banlieues a d'une certaine manière ouvert la voie à la « réislamisation » de la jeunesse « beure » en France, pays qui connaît des difficultés économiques et un racisme croissants (Bloul, 1998: 5). On rappellera aussi que les musulmans, politiquement sous-représentés et perçus comme une menace pour la nation, peuvent être enclins à se tourner vers des "sociétés parallèles ». Les "sociétés musulmanes parallèles" présentes dans les pays d'Europe occidentale (comme la France, l'Allemagne, la Grande-Bretagne, les Pays-Bas et le Danemark), ne sont pas le résultat d'un conservatisme intrinsèque des musulmans, mais leur réaction 
aux mécanismes structurels et politiques d'exclusion. En d'autres termes, la religiosité ne peut être limitée aux croyances des dites minorités. Bien qu'il y ait sans doute des tensions sociales entre les classes, les administrations, l'État, les médias et même les intellectuels, intentionnellement ou pas, font souvent de mauvais diagnostics. Est-ce vraiment leurs différences culturelles, leur refus de l'intégration, leur attitude réactionnaire et leur identité musulmane qui ont fait descendre les musulmans dans les rues? Ou leurs mouvements sociaux d'opposition sont-ils la manifestation d'une résistance contre presque deux siècles de colonialisme, d'exclusion, de racisme, de xénophobie et plus récemment de révolte contre les conditions aggravées de pauvreté ? Les réponses à ces deux questions essentielles fournissent des indices sur la manière dont les individus, les institutions et l'État, abordent le problème. Ceux qui répondent positivement à la première question trouvent l'Islam, culturellement différent et ethniquement divers, «problématique » par nature. On s'attend à ce que "Les Autres » éliminent leurs différences et s'assimilent dans le projet de la civilisation dominante.

On verra dans cet article que l'identité religieuse adoptée par la jeunesse musulmane n'est pas étroitement liée à cette «identité islamiste radicale " si redoutée. Nul besoin de dire que certains jeunes musulmans ont choisi une vue plus fondamentaliste de l'Islam influencée par le Front Islamique du Salut (FIS) qui s'est d'abord organisé en Algérie et a gagné un certain degré de légitimité contre l'ancien État colonial français (Leveau, 1997). Cependant, de récentes études menées en France et dans d'autres pays montrent que les jeunes musulmans considèrent leur identité musulmane d'un point de vue symbolique, que la plupart d'entre aux n'observent pas les rites religieux et qu'ils adoptent une vision du monde de plus en plus laïque et matérialiste (Hargreaves, 1995 ; Tribalat, 1995 ; Kaya et Kentel, 2005). Les travaux menés par Hargreaves (1995 : 120-121) et Tribalat (1995: 96-98) montrent qu'une partie des Nord-Africains en France se considère comme des « musulmans athées ".

7 Il peut y avoir différentes réponses au fait que des tensions sociales et des révoltes se produisent de manière répétée en France. L'objectif de cet article, en nous appuyant sur des études menées en France en 2004, est de soutenir que les récents actes de violence n'étaient pas imprévisibles et qu'ils avaient une base structurelle. En 2004, avec le sociologue Ferhat Kentel, nous avons conduit une étude pour le Centre de Recherche sur les Migrations de l'Université Bilgi d'Istanbul (Kaya et Kentel, 2005). L'étude quantitative a été conduite auprès des immigrés turcs en Allemagne et en France qui ont répondu à un questionnaire de cent items. L'étude qualitative s'est appuyée sur des entretiens approfondis avec des groupes ciblés. L'enquête a concerné environ 1100 personnes en Allemagne et 700 en France et l'on peut considérer les résultats comme représentatifs. Ils montrent que les immigrés en Europe occidentale sont confrontés à d'importants problèmes sociaux structurels. Les processus de désindustrialisation dans toute l'Europe de l'Ouest durant ces deux dernières décennies ont confronté les immigrés au chômage, à la pauvreté et au racisme. Ces travaux ont été complétés par une recherche conduite dans les banlieues parisiennes pendant l'été 2006, où je me suis particulièrement intéressé aux jeunes d'origines africaines.

\section{Bref rappel du processus d'immigration en France}

8 Suite à la Seconde Guerre mondiale, la France s'est trouvée face à un besoin de maind'œuvre et a reçu des travailleurs immigrés du milieu des années 1950 au début des 
années 1970. Une des questions traitée à l'époque, concernait l'apport de population immigrée dans le contexte d'une baisse du taux de natalité. En dissociant le permis de séjour du permis de travail, le gouvernement a ouvert les portes aux travailleurs demandeurs d'emploi et à leur famille; les travailleurs temporaires devenant des résidents permanents (Kivisto, 2002: 172). Bien qu'il n'y ait jamais eu de quotas ethniques, l'administration française a privilégié les migrants en provenance des anciennes colonies et d'Europe. Chronologiquement et en nombre d'immigrés, l'Algérie occupait le premier rang, suivie du Portugal, du Maroc, de l'Italie, de l'Espagne, de la Tunisie puis de la Turquie. À la veille de l'indépendance (1962), 410000 Algériens vivaient en France (Weil, 1996). En 1962, peu de temps après l'indépendance de l'Algérie, les gouvernements français et algérien ont signé un accord de main-d'œuvre, main-d'œuvre qui sera concentrée dans les grandes villes comme Paris, Lyon et Marseille. Le regroupement familial va se développer dès le début des années 1970 et vers 1999, on comptait plus de 700000 Algériens vivant en France avec environ un million de citoyens français d'origine maghrébine (Lanier, 1991) et l'on estimait à 300000 le nombre de Juifs sépharades nord-africains (Morin, 1991). On a aussi estimé que la moitié de la population d'origine nord-africaine vivant en France était berbère, comprenant de nombreux Kabyles, pour partie arabophones et pour partie berbérophones, et que l'autre moitié était d'origine arabe.

Tableau 1 : Origines de la population musulmane de France (estimation globale)

\begin{tabular}{|l|l|l|l|}
\hline Pays d'émigration/origine & Pop. & Pays d'émigration/origine & Pop. \\
\hline Algérie & 1550000 & Turquie & 315000 \\
\hline Maroc & 1000000 & Asiatiques & 100000 \\
\hline Tunisie & 350000 & Demandeurs d'asile et clandestins & 350000 \\
\hline \hline Afrique subsaharienne & 250000 & Autres & 100000 \\
\hline \hline Français convertis & 40000 & & 4155000 \\
\hline \hline Total & & & \\
\hline
\end{tabular}

Source : Ministère de l'Intérieur et Haut Conseil à l'Intégration, L'Islam dans la République, novembre 2000, disponible en ligne : http://lesrapports.ladocumentationfrancaise.fr/BRP/014000017/0000.rtf, accès en novembre 2006.

L'origine nationale ne signifie pas que tous les immigrés d'un pays donné (ex. Turquie ou pays d'Afrique) soient, d'une part musulmans de confession, d'autre part que tous les musulmans de culture soient pratiquants et se reconnaissent dans cette assignation globale.

Bien que la migration de travailleurs ait été suspendue par le gouvernement de Valéry Giscard d'Estaing en 1974, la migration en provenance d'Afrique du Nord et centrale et de Turquie a continué sous les formes du regroupement familial et de l'immigration irrégulière de personnes entrant avec des visas de touristes. Notre récente recherche «Euro-Turcs» montre que $30 \%$ des Turcs de France sont venus en France pour travailler, alors qu'environ $49 \%$ d'entre eux indiquaient que leur mode d'entrée en France était soit le regroupement familial (22\%), soit le mariage (27\%). De la même manière, on estimait que $30 \%$ des Maghrébins sont venus en France comme 
travailleurs et plus de $60 \%$ par regroupement familial (Lloyd, 2003). La migration continue aujourd'hui encore à travers le mariage et la demande d'asile. Le gouvernement conservateur essaya de réduire les effectifs de la population étrangère résidente à l'aide de politiques d'incitations au retour incluant des aides financières. Entre avril 1971 et novembre 1981, le gouvernement français a offert une somme de 10000 francs à chaque immigré chômeur volontaire pour retourner dans son pays d'origine avec sa famille. Bien que cette politique concernât en particulier les États n'appartenant pas à la Communauté Européenne, très peu d'immigrés de pays-tiers ont accepté l'offre. Les immigrés qui ont accepté étaient majoritairement d'origine espagnole ou portugaise. Malgré ces mesures, la population des « étrangers » est restée constante pendant le dernier quart du $\mathrm{xx}^{\mathrm{e}}$ siècle.

\section{La citoyenneté française} et a invité tous les étrangers "amoureux de la Liberté » à se joindre à elle. Le décret d'août 1792 offrait la citoyenneté française à tous les étrangers qui soutenaient la liberté et les principes révolutionnaires par leur action ou leur pensée. La suprématie du principe de jus soli n'a pas changé depuis la Révolution. La loi sur la citoyenneté de 1851 assurait la citoyenneté française à la troisième génération de migrants, tandis que l'amendement de 1889 autorisa la seconde génération à devenir des citoyens de plein droit (Brubaker, 1992: 85-86). Cet amendement a survécu jusqu'à aujourd'hui avec quelques modifications.

11 La loi sur la citoyenneté française inclut deux articles qui solidifient le principe du jus soli : l'article 23 garantit la citoyenneté comme un droit de naissance pour les immigrés de la troisième génération, alors que l'article 44 établit que les immigrés de la seconde génération nés en France et résidants en France depuis l'âge de 13 ans auront la citoyenneté à 18 ans (à leur majorité) à moins qu'ils n'aient choisi une autre citoyenneté ou n'aient commis quelque crime. La loi permet aussi la double nationalité. Cependant elle a été récemment critiquée par les milieux nationalistes qui réclament qu'elle ne fasse pas des étrangers qui ne se sentent pas «Français de cœur» des citoyens sur le papier. La critique nationaliste contre le jus soli a été croissante depuis le début des années 1990, quand le présumé fondamentalisme musulman a commencé à émerger en Occident.

Les Français se voient eux-mêmes comme une nation assimilatrice, mais n'étaient pas préparés à la rapide présence de soulèvements des musulmans et des "personnes de couleur». Alec Hargreaves (1995: 26-27) résume ainsi la situation: «L'invisibilité virtuelle des anciennes générations d'immigrés et de leurs enfants aussi bien que de leurs petitsenfants est perçue comme l'évidence de leur intégration réussie. On sent que les migrants d'après-guerre et particulièrement les migrants des deux dernières décennies menacent cette tradition. C'est devenu une idée commune qu'il est plus difficile d'intégrer des migrants venant $d u$ Tiers Monde, en opposition à ceux qui viennent du reste de l'Europe. Au lieu de se fondre dans la société sans laisser de traces, ils sont en train de devenir plus visibles dans une époque où les gouvernements consécutifs assurent que "l'immigration c'est fini". Ce qui est effrayant, c'est que l'immigration commence à créer des minorités permanentes et diverses dans la société française. » 
13 Pendant un siècle, la France a considéré les immigrés de seconde génération comme des citoyens à part entière. Cependant, l'application du principe du jus soli a subi de sévères attaques venant de l'extrême-droite. Le Front National a ouvertement proclamé : «Être Français, ça se mérite ». Les parties de centre-droit, sous l'influence du Front National, ont proposé le rejet de la citoyenneté française systématique et soulevé la question durant l'année parlementaire 1986. Les immigrés de seconde génération ne se verraient plus accorder la citoyenneté française selon le principe du jus soli; ils devraient la demander et obtenir le consentement de l'État. Le gouvernement Chirac proposa de limiter le principe du jus soli dans le cas des migrants, mais cette proposition rencontra une forte opposition et fut finalement supprimée de l'agenda politique. Une commission défendant l'expansion plutôt que la limitation des droits à la citoyenneté française fût alors constituée (Brubaker, 1992 : 138). Le rapport de la commission devint le fondement de la Loi no 93.933 adoptée le 22 juillet 1993. L'aspect le plus significatif de cette loi concernait le principe de consentement: "toute personne née en France de parents étrangers pourra acquérir la nationalité française sur demande entre l'âge de 16 et 21 ans sous condition de prouver qu'elle a résidé dans le pays les cinq dernières années et n'a pas été condamnée ». Ainsi, ceux qui renoncent explicitement à leur droit de prendre la nationalité française durant l'année qui précède leur majorité ou qui ont été condamnés pour un quelconque délit, ne pourront recevoir la nationalité.

Le processus d'accession à la citoyenneté est secondaire par rapport à celui de l'acquisition de la nationalité. Contrairement à l'acquisition par déclaration, l'accès à la citoyenneté est un choix ouvert, ce qui n'est pas innocent quant au contrôle et à l'approbation par les autorités. La procédure demande cinq années de résidence, d'atteindre l'âge de la majorité, la connaissance de la langue, l'assimilation dans la société française, de n'avoir jamais été condamné à une peine de prison de plus de six mois et de ne jamais avoir commis de crime contre la sécurité de l'État. L'accès à la citoyenneté est basé sur l'assimilation, l'intérêt, l'affection et la loyauté du résident étranger ; c'est ainsi que les dossiers d'étrangers dont la famille vit hors de la France sont en général refusés. On peut dire que le principe d'une création de citoyens politiquement égaux dans la Cinquième République se trouve face à de grands dilemmes qui sont apparus sous de Gaulle et sont toujours d'actualité. La France considère les citoyens comme politiquement égaux sans référence à la religion, à la langue, à la race, à l'ethnicité et au sexe. Elle continue d'ignorer les différences culturelles, religieuses et ethniques mises en valeur par les minorités et poursuit une politique assimilatrice.

\section{Discrimination et dilemme de l'Éducation Nationale}

La discrimination, visible dans la sphère publique, est particulièrement vécue et dénoncée par les musulmans (particulièrement les Nord-Africains). En outre l'Éducation Nationale désignée pour produire des citoyens français et défendre une Nation française homogène a fait l'objet de nombreuses critiques. Ainsi L'étude PISA (Programme for International Student Assessment), conduite annuellement dans les divers pays de l'OCDE depuis 2001, montre que la qualité et les opportunités données par l'éducation sont significativement basses dans les quartiers peuplés par des populations minoritaires, que la violence scolaire y est un problème important et que les écoles y reçoivent un investissement technologique inadéquat. 
16 Tribalat (2003) montre que le taux d'illettrisme est supérieur à la moyenne parmi les populations d'origine algérienne et marocaine. Le sociologue François Dubet quant à lui observe que : "Les relations dans les écoles, comme les relations sociales dans leur totalité, deviennent de plus en plus racistes. Les individus sont perçus et marqués comme porteurs d'identités ethniques". Pour le dire simplement, alors que les écoles définissaient dans le passé certains élèves comme des enfants de la classe ouvrière, elles les définissent maintenant comme des enfants d'immigrés. Si les problèmes d'un enfant étaient attribués à la pauvreté de ses parents autrefois, ils sont aujourd'hui attribués au fait que ses parents sont des immigrés, même lorsque cet enfant appartient à la troisième génération. Le comportement des garçons aurait été décrit comme "hostile" autrefois, il est aujourd'hui décrit comme "ethnique" ». (Dubet, cité par Starkey, $2003: 120$ ).

17 Avec la racialisation du discours et la faillite ou du moins les difficultés du système éducatif, on aboutit au constat que pratiquement aucun musulman n'a aujourd'hui obtenu un siège à l'Assemblée Nationale. La combinaison de l'absence de modèles de réussite avec le marquage raciste fait qu'il est difficile pour les enfants issus de minorités ethniques de s'identifier au républicanisme qui ne réussit pas à leur donner le moindre capital pour accéder à des postes valorisés et valorisants. Et pourtant l'éducation citoyenne au travers du système scolaire a toujours été considérée comme l'outil d'intégration des groupes divers à l'intérieur d'un système éducatif unitaire qui était défini comme républicain. Les valeurs universelles et libérales ont toujours primé sur les identités culturelles, ethniques ou religieuses. Mais on peut de se demander à la lumière de nos enquêtes si cela ne produit pas une société française à laquelle n'adhèrent plus les enfants d'immigrés.

18 En cela nos données viennent renforcer les résultats de Tribalat sur la discrimination subie par les immigrés et les étrangers. Les Turcs de France estiment que la discrimination est leur principal problème (17\%). Comme l'explique un de nos enquêtés: "Aucun pays ne pouvait soutenir la comparaison avec la France jusqu'à 1985. Mais nous sommes maintenant face à un problème racial. Ce n'est pas seulement Le Pen; les communistes voient la situation dans la même perspective ». (Kaya et Kentel, 2005 : 33). Ainsi comme l'écrit Tribalat (2003) : «Si je subis une discrimination, pourquoi travaillerais-je dur à l'école?». Tribalat affirme que la discrimination pose un problème d'incohérence entre l'idéal républicain et la réalité vécue par la société française. On constate que le taux de chômage parmi les diplômés de l'Enseignement supérieur oscille entre 5 et $27 \%$ selon leurs origines: française autochtone ou nord-africaine ${ }^{7}$. La discrimination n'est pas seulement limitée à la sphère scolaire, elle va bien au-delà. Jusqu'à récemment, parler de la discrimination était tabou car ressenti comme une menace pour l'ordre républicain (Tribalat, 2003 : 135). Les écrits de Pierre Sadran (2003: 53) montrent l'existence d'une crise de légitimité dans l'idéologie française du républicanisme : «Les Français ont commencé à perdre leur croyance dans le fait d'avoir été "privilégiés". Premièrement et surtout, le pays a été secoué par le bas comme par le haut par la stagnation de l'État-nation, la décentralisation et les dynamiques de l'intégration européenne. Deuxièmement, l'universalisme à la française devrait abandonner ses ambitions à représenter une certaine adaptation de l'universalisme et se contenter d'être la représentation d'un universalisme spécifique... Enfin, le modèle français d'intégration construit sur l'école ne fonctionne plus comme par le passé. La Coupe du Monde de 1998 gagnée par la France a rassemblé les différentes cultures de France, non sur les couleurs bleu-blanc-rouge du drapeau national, mais sur les couleurs black-blanc-beur des joueurs de l'équipe nationale. " 

Lor nos enquêtes nous avons constaté que les chômeurs que l'on rencontrait dans des quartiers comme Kreuzberg (Berlin), Keupstrasse (Cologne), Villiers-le-Bel, La Courneuve, Saint-Denis ou Créteil (région parisienne) étaient réellement désavantagés ${ }^{8}$. Ainsi si l'on se réfère au taux de chômage des jeunes Turcs de Berlin, il s'élève à $40 \%$ et celui «Beurs» en France à $42 \%$. Ces chiffres sont très élevés, comparés aux taux moyens de chômage des populations globales (approximativement de l'ordre de 12 \%). L'exclusion qu'ils subissent et les difficultés dans lesquelles ils se trouvent les conduits à des situations d'isolement et de marginalisation, voire "d'hyper marginalisation » dans certains quartiers. Les musulmans dans les banlieues des métropoles fréquentent « leurs » écoles isolées, prient dans « leurs » mosquées, font leurs courses dans « leurs » boutiques et développent "leur » économie en marge de la société globale. Le départ des membres de la classe moyenne musulmane notamment vers des quartiers mieux intégrés a laissé sur place les plus pauvres des pauvres, de plus en plus en dehors de l'économie urbaine et privés des supports institutionnels qui leur permettraient une vie plus décente. Alors que la production industrielle est en déclin, ces populations ne peuvent s'adapter aux mutations économiques en cours et tombent dans un état de chômage chronique, de précarité et d'exclusion sociale, sinon de solitude (Kivisto, 2002).

23 Ce qui pourrait le mieux décrire le point de vue de ces populations «hypermarginalisées » est le «nihilisme », ou encore l'« anarchisme ». Religion et ethnicité offrent des «solutions » attractives pour des personnes enfermées dans des problèmes inextricables. Il n'est guère surprenant, pour des gens ayant une vue morose et une

Revue européenne des migrations internationales, vol. 22 - n³| 2006 
vision négative de leur avenir, qui ne tirent aucun bénéfice de la société dans laquelle ils vivent et qui sont mises en marge du capitalisme global, qu'elles se reposent sur des valeurs religieuses ou ethniques, valeurs dont elles croient ne pas pouvoir être dessaisies.

Dès lors face à ces diagnostics et devant le caractère "islamique » souvent invoqué par les jeunes immigrés musulmans dans les actes de violence si l'analyse n'est pas menée avec rigueur, elle ne peut que conduire à reproduire la thèse du «choc des civilisations». Il est donc important de souligner que l'«identité islamique » utilisée par les jeunes, qui affiche ainsi sa résistance au système de différentes manières (musique, tags et graffiti, danse, pillage, incendie...), en France comme ailleurs en Europe, n'est ni essentialiste ni radicale. La référence islamique utilisée dans des actes d'opposition est principalement l'expression d'un désir de se revendiquer d'un discours général légitimé et contre-hégémonique, comme peut l'être celui de l'islam dans le monde actuel, et d'en tirer ainsi un pouvoir symbolique'. Il semble qu'aujourd'hui la religion vienne souvent remplacer l'absence d'un mouvement gauchiste. Michel de Certeau (1984: 183) nous rappelle les similarités entre le discours religieux et la gauche: la religion offre un monde différent et alternatif et autorise un avenir différent. On se souviendra aussi que les actes de violence qui se sont produits à Paris se sont rapidement étendus à d'autres cités et pays, nous autorisant à penser qu'il s'agit là d'une manifestation de la solidarité entre les éléments d'une diaspora islamique.

L'islam est perçu par les Occidentaux comme une menace pour le mode de vie européen. Le fondamentalisme islamique est décrit comme la source de comportements xénophobes, racistes et violents. Cependant, en renversant ce point de vue, la montée des valeurs religieuses peut être interprétée comme la résultante de problèmes sociaux comme la pauvreté, le chômage, le racisme, la xénophobie, l'isolement, l'absence de représentation politique et la menace d'une assimilation sans contrepartie. Pour se défendre face à ces défis, les discours sur la culture, l'identité, la religion, l'ethnicité ou les traditions et le passé ont acquis valeur de stratégie de survie pour les minorités en général et les immigrés en particulier. En reconstruisant un passé prestigieux et en insistant sur la culture d'origine, ethnicité et religion semblent fournir une double référence pour les communautés immigrées :

- comme une forme de reconstruction du contemporain, sans analyse critique du statu quo d'un passé « glorieux » et mythifié, la culture authentique. L'ethnicité et la religion sont utilisées par des sujets vivant en situation diasporique en tant qu'instruments stratégiques permettant de résister à l'exclusion, à la pauvreté, au racisme et à la discrimination institutionnelle.

- secondairement, comme une façon de donner à un individu le sentiment de l'indépendance face aux critères imposés par la société majoritaire, parce que le passé, les traditions, la culture et la religion symbolisent des valeurs et des croyances que les sujets vivant en diaspora pensent faire partie de leur être profond.

Dans ce contexte, les opinions d'Herbert Gans sur l'ethnicité symbolique et la religiosité sont très éclairantes. Selon Gans, ethnicité symbolique et religiosité sont des ressources accessibles à ceux qui veulent se garder de l'essentialisme ethnique ou religieux, bien que se réservant la possibilité d'expérimenter cette ethnicité ou ce sentiment religieux de temps en temps (Gans, 1979 : 1-2). L'adoption de ces valeurs par les plus jeunes des «Turcs de France » ou des «Africains de France» comme source de l'identité est une attitude contraire à l'essentialisme. Il s'agit d'un processus d'identification, que Gans 
définit comme une "ethnicité symbolique» ou ce que j'appelle une "religiosité symbolique ». Le processus montre que ce qui est essentiellement religieux devient un marqueur ethnique et que "ethnicité " et « religiosité " sont les deux composantes principales d'une identité politique développée par des minorités confrontées à la menace d'un isolement structurel imposé par la société majoritaire. La citation suivante, tirée de Gans (1979:6) résume clairement ce processus : «Alors même que les cultures ethniques et les groupes deviennent moins fonctionnels et que l'identité devient le premier moyen d'être ethnique [et religieux], l'ethnicité [et la religiosité] gagne une fonction plus symbolique qu'instrumentale et perd de son importance dans la vie familiale, devient une activité de loisirs. Le comportement symbolique peut se manifester de différentes manières, mais inclut fréquemment l'usage de symboles. Les symboles ethniques sont surtout des pratiques culturelles individuelles qui ont été acquises des cultures ethniques les plus anciennes; ils sont abstraits de cette culture et rayés de leurs significations originales. »

Cette citation nous donne une idée du contexte dans lequel le concept de «musulman athée » est utilisé par de jeunes Nord-africains pour se définir pendant et avant les récentes émeutes. Si la religiosité et l'athéisme sont deux concepts opposés, le qualificatif de "musulman » précédant celui "d'athée » doit porter une signification autre que celle du registre de la religiosité. L'adjectif «musulman » utilisé dans cette expression est une référence de ce qui en va de l'ethnicité. Même ici, l'usage de l'islam exprime la résistance et l'opposition de la minorité contre la majorité et l'officialité. Ressentant qu'ils sont considérés avec des préjugés par la majorité de la population parmi laquelle ils vivent, les groupes minoritaires sont enclins à exagérer les traits qu'ils croient poser problème à cette majorité durant leur processus d'identification ; c'est ainsi qu'ils vont souligner leur appartenance à l'islam et les concepts musulmans, qu'ils pensent nécessairement poser question à la majorité chrétienne, lorsqu'ils expriment leur identité. Les concepts d'«islam» et de «musulman» renvoient politiquement et économiquement à des limites construites entre la majorité et les minorités et par conséquent portent un caractère ethnicisant.

28 La religion est l'une des sources importantes de la formation de l'identité diasporique. Ainsi, elle n'a pas de signification essentialiste, mais bien plus une signification symbolique pour les générations issues de l'immigration et au-delà pour la diaspora. La signification du fait religieux pour la jeunesse est liée au fait que ces jeunes sont perçus de manière préjudiciable par la majorité. Cette majorité autochtone utilise l'islam comme le point focal d'une identité définissant des groupes aussi divers que des jeunes originaires d'Afrique du Nord, du Pakistan, du Bengladesh ou de Turquie. En réaction, nombreux sont ceux qui développent une loyauté symbolique envers la religion de leurs parents. Un bon exemple de ce processus peut être donné par les jeunes turcs berlinois. Ainsi, un groupe de rap germano-turc basé à Berlin dans les années 1990 expliquait le choix d'Islamic Force comme nom de scène par le fait qu'il voulait protester contre la représentation négative de l'islam parmi la population allemande et pensait qu'il devait la provoquer. De façon tout à fait intéressante, Islamic Force était un groupe de rappeurs utilisant un langage d'abord et surtout universaliste, en totale opposition avec le nom choisi (Kaya, 2001). Steven Vertovec (1995) qualifie cette expression de l'identité comme "l'identité culturelle islamique». Il y a des similarités significatives entre les jeunes immigrés musulmans asiatiques vivant dans la ville de Keighley dans le nord de l'Angleterre et se définissant comme des "jeunes musulmans", la jeunesse turque berlinoise ou la jeunesse maghrébine de France. Ces quelques exemples nous 
montrent que ces identités culturelles et diasporiques émergent à l'intérieur de processus de relations dialectiques et dialogiques entre une majorité et des minorités. C'est un processus de sécularisation de l'islam en diaspora, par lequel le sentiment religieux s'individualise, se privatise, face aux besoins émergents d'individus qui subissent les impacts collectifs d'une étrangeté qui perdure. Par conséquent, l'espace islamique devient un espace dans lequel les "post-migrants " (les enfants d'immigrés) et les «trans-migrants » sont en quête de reconnaissance. L'allégeance islamique peut aussi être interprétée pour ces jeunes comme une quête d'émancipation face à la culture parentale emprisonnant la religion dans "leur culture authentique». Cependant, l'allégeance de cette jeunesse post-migrante envers l'islam n'est pas limitée à celui du pays d'origine, mais s'étend à l'Umma, communauté musulmane internationale "recherchant la solidarité avec et dans l'intérêt de luttes comme celles de la cause palestinienne et les conflits en Bosnie, Tchétchénie, Afghanistan, Irak et Liban » (Cesari, 2003). On peut donc avancer l'idée que la jeunesse diasporique se trouve affiliée à un islam bien plus symbolique que véritablement politique. C'est une position intermédiaire entre le sentiment religieux et l'action politique ${ }^{10}$.

\section{Conclusion}

Les récentes émeutes observées dans la banlieue parisienne expriment la réaction d'une jeunesse française musulmane, surtout d'origines maghrébines et africaines, face à des questions sociales objectives telles que : chômage, pauvreté, manque d'éducation, inégalités, racisme et xénophobie, assimilation, solitude, isolement et exclusion sociales. Mais cette résistance est aussi une indication du fait que le mythe du citoyen politiquement égal à l'intérieur de la tradition républicaine française se porte mal. La tentative de construction d'un "citoyen politiquement égal » s'est manifestée comme celle d'un citoyen qui n'a pas réussi complètement à atteindre cet objectif de droits politiques pleins et entiers. Le fait qu'aucun député de confession musulmane ne siège à l'Assemblée Nationale, malgré la présence d'au moins cinq millions de musulmans dans la Nation française, semble l'illustrer. Le fait aussi que ces citoyens d'origines musulmanes soient encore rares dans les instances démocratiques locales devient un véritable dilemme pour le fonctionnement de la démocratie française. Ce dilemme est le produit d'une erreur d'interprétation des développements sociaux récents que connaît la société française par les élites politiques, élites qui ont fermé les yeux sur l'existence de la diversité et qui ont été séduites depuis bien longtemps par le mythe selon lequel tous les citoyens français sont égaux sans référence aucune à l'appartenance religieuse, à la diversité linguistique, aux appartenances raciales, ethniques, de classe ou de genre ${ }^{11}$.

Alors que la société subit un processus de désindustrialisation et de croissance des inégalités politiques, sociales, éducatives et professionnelles, et que ces phénomènes aliènent les immigrés face au projet républicain, la tendance générale est à stigmatiser la religion, l'ethnicité, la langue et la tradition, quoi que l'on puisse en retirer de positif, même limité ou contrôlé. L'émergence d'organisations sociales parallèles est automatiquement mal comprise par la majorité et attribuée à un conservatisme consubstantiel de l'islam et à une résistance intrinsèque des immigrés musulmans. Comme résultante, le fait que la formation de sociétés parallèles soit liée à des questions comme le chômage, la désindustrialisation, l'isolement et le racisme, reste 
traditionnellement largement ignoré ou sous-estimé. Les élites conservatrices, à l'instar de Nicolas Sarkozy, cèdent délibérément aux mauvaises interprétations, ce qui entraîne le sentiment que les immigrés sont de potentiels « ennemis de l'intérieur » devant être éliminés. Avec cette représentation problématique, à la fois des immigrés et des musulmans, dans les médias, le problème tourne à l'impasse. Si l'on agglomère interprétations fallacieuses et erreurs de jugement, il est facile de voir comment on peut passer doucement d'une image de "voisins de palier» à celle "d'ennemis de l'intérieur".

31 L'alarme sonne pour la Cinquième République Française. Le caractère assimilationniste qui veut ignorer les différences, l'incapacité à transférer des droits politiques de la théorie à la pratique, la centralisation de l'État et la structure démographique du corps parlementaire, donnent prise à une opposition sociale bloquée dans ses expressions. Certes important pour les immigrés, le projet républicain est également crucial pour les Français dans leur ensemble. Les récentes normes européennes, imposées au droit national, tendent à promouvoir le respect de la décentralisation de la gouvernance et la participation politique des minorités. L'élection de deux députés européens français d'origine musulmane au Parlement européen, la création en 2003 d'un Conseil consultatif du Culte Musulman par le ministre de l'Intérieur issu de l'UMP (Union pour un Mouvement Populaire) sont autant d'indications importantes d'évolutions. Ces changements institutionnels correspondent à la transformation de la définition jacobine de la République, qui insistait sur le fait qu'il ne pouvait y avoir d'intermédiaires entre l'individu et l'état, et rejetait toutes les instances médiatrices comme les provinces, les communautés ethniques, les syndicats ou les Églises (Safran, 2003 : 54). À côté de ces changements institutionnels, on doit souligner que le langage islamique utilisé par les jeunes immigrés musulmans est de caractère symbolique. C'est en fait un langage ethnoculturel utilisé par des masses sécularisées et laïcisées. On peut noter que des groupes marginaux qui ne peuvent entrer sur la scène politique au travers de canaux légitimes font usage de discours religieux ou ethniques. Le discours en usage chez les Beurs de France est, de ce point de vue, l'expression de cette quête politique. De très nombreux musulmans peuvent trouver leur place dans les équipes sportives nationales, la culture hip-hop, le cinéma, les arts plastiques et beaucoup d'autres champs d'expression publique, mais sans obtenir la moindre place dans l'arène politique. Vues ainsi, les évènements vécus en France sont des actions revendicatives d'immigrés à la recherche d'une reconnaissance politique et d'égalité. Il ne sera pas surprenant, et ne peut être considéré comme une simple coïncidence que la France, pays d'origine des mouvements de 1968 soit aussi celui de la naissance de mouvements d'immigrés, qui ont véritablement montré leur allégeance aux quatre piliers de la rhétorique républicaine en liant leur recherche à «Liberté, Égalité, Fraternité et Laïcité ». 


\section{BIBLIOGRAPHIE}

BLOUL Rachel A. D (1998) From Moral Protest to Religious Politics : Ethical Demands and Beur Political Action in France, The Australian Journal of Democracy, (9) 1, pp. 11-30.

BLOUL Rachel A. D (1999) Beyond Ethnic Identity : Resisting Exclusionary Identification, Social Identities, (5) 1, pp. 7-30.

BODY-GENDROT Sophie (2002) Living Apart or Together with our differences ? French Cities at a Crossroads, Ethnicities, (2) 3, pp. 367-385.

BRUBAKER Rogers (1992) Citizenship and Nationhood in France and Germany, Cambridge, Harvard University Press.

CESARI Joselyne (2003) Muslim Minorities in Europe : The Silent Revolution, in J. Esposito and F. Burgat (Eds.), Modernizing Islam : Religion in the Public Sphere in the Middle East and in Europe, New Jersey, Rutgers University Press, pp. 251-269.

CERTEAU Michel de (1984) The Practice of Everyday Life (translated by Steven Rendall), Berkeley, University of California Press.

FETZER Joel S. and J. Christopher SOPER (2005) Muslims and the State in Britain, France and Germany, Cambridge, Cambridge University Press.

GANS Herbert (1979) Symbolic ethnicity : The future of ethnic groups and cultures in America, Ethnic and Racial Studies, (2) 1, pp. 1-20.

HARGREAVES Alec G. (1995) Migration, Race and Ethnicity in Contemporary France, London, Routledge.

JELEN Brigitte (2005) « Leur Histoire est notre Histoire » : Immigrant Culture in France between Visibility and Invisibility, French Politics, Culture and Society, (23) 2, pp. 101-125.

KAYA Ayhan (2001) Constructing Diasporas : Turkish Hip-Hop Youth in Berlin, Bielefeld, Transcript.

KAYA Ayhan and Ferhat KENTEL (2005) Euro-Turks : A Bridge, or a Brewach, between Turkey and the European Union? Brussels, Centre for European Policy Studies.

KIVISTO Peter (2002) Multiculturalism in a Global Society, Oxford, Blackwell.

LANIER Pierre (1991) Les nouveaux visages de l'immigration, Lyon, Chronique Sociale.

LEVEAU Rémy (1997) The Political Culture of the "Beurs", in Steven Vertovec and Ceri Peach (Eds.), Islam in Europe : The Politics of Religion and Community, London, MacMillan, pp. 147-155.

LLOYD Cathie (2003) Multi-causal Conflict in Algeria : National Identity, Inequality and Political Islam, QEH Working Paper Series - QEHWPS104 (April).

MAFFESOLI Michel (1996) The Time of the Tribes, Cambridge, Cambridge, Polity Press.

MACINTYRE Alasdair (1971) Against the Self-Images of the Age : Essays on Ideology, New York, Schoken Books.

MORIN Georges (1991) La mosaïque des Français du Maghreb et des Maghrébins de France, in L. C and Y. Lacoste (Éds.), L'État du Maghreb, Paris, La Découverte. 
NOIRIEL Gérard (1988) Le creuset français. Histoire de l'immigration $\mathrm{xIX}^{\mathrm{e}}-\mathrm{xx}^{\mathrm{e}}$ siècles, Paris, Le Seuil.

SADRAN Pierre (2003) The French State and the Challenges of the Twenty-First Century, in Susan Milner and Nick Parsons (Eds.), Reinventing France : State and Society in the Twenty-First Century, Hampshire, Palgrave, pp. 49-62.

SAFRAN William (2003) Religion and Laïcité in a Jacobin Republic : The Case of France, in William Safran (Éd.), The Secular and the Sacred : Nation, Religion and Politics, London, Frank Cass, pp.54-81.

SILVERSTEIN Paul and TETREAULT Chantal (2005) Urban Violence in France, Middle East Report Online, (November), http://www.merip.org

STARKEY Hugh (2003) Education for Citizenship : Reinventing the French Republic, in Susan Milner and Nick Parsons (Eds.), Reinventing France : State and Society in the Twenty-First Century, Hampshire, Palgrave, pp. 110-126.

TRIBALAT Michèle (1995) Faire France : Une enquête sur les immigrés et leurs enfants, Paris, Éditions La Découverte.

TRIBALAT Michèle (2003) The French "Melting Pot": Outdated - or in need of reinvention ? in S. Milner and N. Parsons (Éds.), Reinventing France : State and Society in the Twenty-First Century, Hampshire, Palgrave, pp. 127-142.

VERTOVEC Steven (1995) Young Muslims in Keighley, West Yorkshire: Cultural identity, context and “community”, Unpublished Research Paper. Coventry, Centre for Research in Ethnic Relations, University of Warwick.

WEIL Patrick (1996) Pour une nouvelle politique d'immigration, Esprit, (avril 1996), pp. 136-154.

ZUBAIDA Sami (2003) Islam in Europe, Critical Quarterly, (45) 1-2, pp. 88-98.

\section{NOTES}

1. Cité par Silverstein er Tétrault, Urban Violence in France, Middle East Report Online, http:// merip.org

2. En réalité, ils étaient trois : Ziya et Bouna sont décédés, Mühittin, assez gravement brûlé, a été hospitalisé (NdlR).

3. La Police a effectué une enquête sur les causes des évènements et a rapidement conclu à l'absence de connexion ou de relation entre ces émeutes et une quelconque organisation terroriste internationale.

4. Il n'existe aucun chiffre officiel en France sur la répartition de la population selon les confessions ou les groupes ethniques, seule la nationalité est prise en compte.

5. L'anglais avec insider et outsider dispose de deux termes, difficilement traduisibles en français. Andrea Rea et Maryse Tripier proposent «établi » pour insider et gardent le terme outsider (Andrea REA et Maryse TRIPIER, Sociologie de l'immigration, Paris, la Découverte, coll. Repères, $2003: 81$ ). Insider pourrait être également traduit par « initié ». NdlR.

6. Cette hypothèse a été significativement renforcée par une récente étude sur le terrain conduite à Paris durant l'été 2006. Bien qu'il semble que ce glissement discursif soit plutôt une construction intellectuelle, il a connu une très rapide publicité. Il est possible de trouver plusieurs blogs ou interventions sur le réseau Internet, concernant directement la circulation de ce discours radical.

7. Afin de contourner le racisme institutionnel expérimenté sur le marché du travail comme dans d'autres sphères de la vie publique et sociale, les jeunes issus de l'immigration tendent à donner 
des prénoms français à leurs enfants. Gérard Noiriel indique que cette pratique est en réalité plutôt ancienne parmi les immigrés : dans une communauté polonaise du nord de la France, les taux étaient de $44 \%$ en 1935, 73 \% en 1945, 82 \% en 1982 et $98 \%$ en 1960 (Noiriel, $1998: 233$ ).

8. Beaucoup de ces quartiers observés durant nos séjours et lors de nos recherches ont en commun d'être qualifiés d'enclaves turques. En réalité, ces enclaves ethniques sont rares. Voir Kaya et Kentel (2005). La Courneuve, Saint-Denis et Créteil ont été inclus dans la liste à la suite du travail de terrain effectué dans les banlieues de Paris durant l'été 2006.

9. Pour une discussion plus approfondie de cette idée, voir Zubaida (2003).

10. Comme le mentionne à juste titre Jocelyne Cesari, on ne peut cependant nier que certains se tournent vers la sécularisation tandis que d'autres optent pour le fondamentalisme dans cette position politique intermédiaire.

11. On peut remarquer la particularité du Parlement français en matière de composition des membres des deux Assemblées face aux autres pays de l'UE. Ceci est marqué aussi bien par la moyenne d'âge des parlementaires que par l'absence de parité entre sexes ou la présence de représentants issus des récentes immigrations (alors que la moyenne d'âge des parlementaires français est de 57 ans, elle est de 50 ans à la Chambre des Communes britannique et de 49 ans au Bundestag allemand en 2005.

\section{RÉSUMÉS}

Il est plus plausible d'interpréter les mouvements dans les banlieues, en 2005, ainsi que les précédents, non pas comme une série d'émeutes anti républicaines, mais plutôt comme un jugement critique du républicanisme concret et conservateur tel qu'il est pratiqué actuellement. Le langage populaire des émeutes n'a jamais été surtout religieux, ethnique ou centrifuge. Le langage de ces émeutes à répétitions depuis le début des années 1990 est plutôt l'expression de la pauvreté, de la violence et de l'exclusion. À la vérité, ce langage découle d'une vraie rhétorique républicaine, qui tente de dénoncer la pauvreté, le chômage, la xénophobie, les tensions urbaines, la politique économique néo libérale et la violence qui prédominent dans les banlieues exclues socialement et politiquement. La crise de l'éducation républicaine, qui reposait sur l'objectif de former des citoyens égaux et politiquement conscients, souligne le fait que les cloches sonnent pour la cinquième République en France. Le présent papier a pour objectif de montrer comment les Musulmans français originaires du Turquie et d'Afrique du Nord conçoivent les inégalités politiques, sociales et économiques actuelles.

French-Muslims: Survey of a Republican Rebellion. It is more plausible to interpret the 2005 banlieue movements and the preceding ones not as a set of anti-republican riots, but rather as a critical assessment of existing real and conservative republicanism. The popular language of the riots has never essentially been religious, ethnic or centrifugual. The language of these ongoing riots since early 1990s is rather the expression of poverty, violence and exclusion. This language indeed derives from quite a republicanist rhetoric, trying to denounce poverty, unemployment, xenophobia, urban tension, neo-liberal political economy and violence prevailing in the socially and politically excluded banlieues. The crisis of the republicanist education, which was based on the objective of forming equal and political citizen highlights the fact that the bells ring for the Fifth French Republic. This work aims to reveal the ways in which French-Muslims of migrant 
origin coming from Turkey and North Africa conceive of the existing political, social and economic inequalities.

Los franceses musulmanes: encuesta sobre una rebelíon republicana. Es más plausible interpretar los movimientos de las «banlieues» (barrios periféricos) de $2005 \mathrm{y}$ de otros precedentes no como una serie de insurrecciones antirrepublicanas sino como un juicio crítico del republicanismo concreto y conservador practicado actualmente. El lenguaje popular de las insurrecciones nunca ha sido religioso, étnico o centrífugo. El lenguaje de estas insurrecciones a repetición desde los años 1990 es más bien la expresión de la pobreza, de la violencia y de la exclusión. Este lenguaje deriva de una verdadera retórica republicana et intenta denunciar la pobreza, el desempleo, la xenofobia, las tensiones urbanas, la política económica neo-liberal y la violencia predominantes en las «banlieues» social y políticamente excluidas. La crisis de la educación republicana cuyo objetivo era el de formar ciudadanos iguales y políticamente conscientes, subraya el hecho de que las "campanas suenan" para la Quinta Republica francesa. El objetivo de este artículo es mostrar cómo los musulmanes franceses originarios de Turquía o del Norte de África conciben las desigualdades políticas, sociales y económicas actuales.

INDEX

Mots-clés : générations issues de l'immigration, identité religieuse, islam, musulmans, Turcs Index géographique : France

\section{AUTEURS}

\section{AYHAN KAYA}

Professeur à l'Université Bilgi d'Istanbul, Directeur du Centre de Recherche sur les Migrations. Istanbul Bilgi University, Center for Migration Research, Inönü Cad. n²8, Kuştepe, TR 34387 şişli, ISTANBUL ayhank@bilgi.edu.tr 